\title{
Effect of nerve monitoring on complications of thyroid surgery
}

\author{
Suleyman Demiryas, ${ }^{1}$ Turgut Donmez, ${ }^{2}$ Erdinc Cekic ${ }^{3}$ \\ ${ }^{1}$ Department of General Surgery, Istanbul University, Cerrahpasa Faculty of Medicine, Istanbul, Turkey \\ ${ }^{2}$ Department of General Surgery, Lutfiye Nuri Burat State Hospital, Istanbul, Turkey \\ ${ }^{3}$ Department of Otolaryngology Surgery, Lutfiye Nuri Burat State Hospital, Istanbul, Turkey
}

\begin{abstract}
OBJECTIVE: The most frequent and critical complications of thyroid surgery are recurrent laryngeal nerve palsy and hypocalcemia. In first years of the 21st century, intraoperative neuromonitoring (IONM) was introduced as a new technique to avoid the recurrent laryngeal nerve (RLN) injury. However, the role and the benefits of IONM are still controversial. In this study, we aimed to compare the postoperative complication rates during thyroid surgery with IONM usage (Group 1) and conventional direct visual technique without IONM usage (Group 2).
\end{abstract}

METHODS: We conducted retrospective review of all patients undergoing thyroid surgery in Lütfiye Nuri Burat State Hospital General Surgery Department between 2014 and 2016 years. Patients have been classified in to two groups: Group 1 and 2.

RESULTS: Overall, 191 patients were included in the study; Group 1 comprised 79 patients and Group 2 comprised 112 patients. Unilateral RLN paralysis was observed in 7 patients in Group 1 (8.9\%) and 15 patients in Group 2 (13.4\%) without any significant difference between the groups $(p=0.368)$. Hypocalcemia was encountered in 5 patients $(6.3 \%)$ in Group 1 and 18 patients $(16.1 \%)$ in Group 2; this difference was statistically significant $(p=0.045)$. Other complications (such as hematoma and suture reaction) were not significantly different. Operation time was found to be significantly shorter in Group 1 (Mean time, $93.08 \mathrm{~min}$ ) than in Group 2 (116.54 $\mathrm{min})(\mathrm{p}=0.03)$.

CONCLUSION: Proven effect of IONM on RLN paralysis is still controversial. However, easy identification of RLN, which gives more confidence to surgeon, and shorter operation time may be factors to lower hypocalcemia rates.

Keywords: Hypocalcemia; intraoperative neuromonitoring; nerve identification; recurrent laryngeal nerve injuries; thyroidectomy.

$\mathrm{T}$ he most frequent and critical complications of thyroid surgery are recurrent laryngeal nerve palsy and hypocalcemia [1]. Malignancy, Graves' Disease, huge size multinodular goiter, reoperative surgery, urgent reoperation due to bleeding, central compartment neck dissection, prolonged surgery, and inadequacy of experience of surgeon are the factors related to complication risk increment in thyroid surgery [1-6].

The best and most acceptable technique to avoid RLN (Recurrent laryngeal nerve) injury the identifica- tion of RLN during surgery $[3,7]$. To avoid the RLN injury and increase the comfort and confidence of surgeon during operation, intraoperative neuromonitoring (IONM) was introduced as a new technique in addition to direct visual technique in first years of the 21 st century $[3,7,8]$. IONM helps the surgeon to identify and verify the functional integrity of the $\operatorname{RLN}[9,10]$. However, unfortunately the role and benefits of IONM are still controversial [11].

RLN injury was resulted with vocal cord paralysis,

Received: October 04, 2017 Accepted: October 09, 2017 Online: January 19, 2018

Correspondence: Dr. Suleyman DEMİRYAS. Istanbul Universitesi, Cerrahpasa Tip Fakultesi, Genel Cerrahi Anabilim Dali, Istanbul, Turkey. Tel: +90 2124143000 e-mail: suleyman.demiryas@istanbul.edu.tr

(c) Copyright 2018 by Istanbul Provincial Directorate of Health - Available online at www.northclinist.com 
which may be transient or permanent. This impairment in cord mobility causes postoperative dyspnea, dysphagia, and dysphonia. The type of the injury (such as heat, compression, stripping, neuritis, cut, or difficult intubation) is important in predicting the progression of symptoms. Generally, traumas due to vocal cord or RLN edema result with transient palsy $[1,2]$.

In case of an absence of an accepted consensus regarding IONM usage, the guideline of the American Academy of Otolaryngology Head and Neck Surgery advise IONM usage during thyroid surgery for voice quality preservation $[1,3,12]$. Both the German Association of Endocrine Surgeons and the International Neural Monitoring Study Group advise the IONM usage in all thyroid surgeries $[13,14]$, but the American Head and Neck Society advises its usage only for patients with thyroid cancer.

In this study, we aimed to compare the postoperative complication rates during thyroid surgery with IONM usage and conventional direct visual technique without IONM usage.

\section{MATERIALS AND METHODS}

\section{Patients and groups}

This retrospective study was approved with local Ethic Committee of Haseki Training and Research Hospital, Istanbul-Turkey (Date/No:10.08.2016/366). Retrospective review of all patients undergoing thyroid surgery in Lütfiye Nuri Burat State Hospital General Surgery Department in 2014-2017 was conducted. Patients have been classified in to two groups. Patients operated with IONM were classified as Group 1 (IONM group); patients operated with conventional direct visual technique without IONM were classified as Group 2 (Non-IONM group). All patients were preoperatively and postoperatively examined for vocal cord mobility as a routine by an otolaryngologist in the ENT (Ear Nose Throat) Department with flexible fiberoptic laryngocscope. Inclusion criteria were multinodular or nodular goiter; Graves' disease; thyroid cancer; male and female patients aged 18-80 years; and ASA scores I, II, and III. Exclusion criteria were pregnancy, patients aged less than 18 years, preoperative laryngeal surgical history, preoperative vocal cord pathology, and radiation history to neck.

All patients were operated in a single department with an experienced surgeon. Preoperative hospital records, age, sex, Body Mass Index (BMI, $\left.\mathrm{kg} / \mathrm{m}^{2}\right)$, comorbidities, laboratory results, ultrasonographic findings, and ENT consultation notes were retrospectively recorded. Surgery type, IONM usage, parathyroids situation, RLN visualization, drainage usage, operation time were recorded intraoperatively. Duration of hospital stay, complications, pathological results, and postoperative vocal cord mobility were recorded postoperatively.

Retrospective review of all 212 patients undergoing thyroid surgery in 2014-2016 was conducted. Of them, complete operative and follow-up data were found on 191 patients.

All patients' postoperative calcium levels were measured first at the 8th hour. Calcium levels less than 8.5 $\mathrm{mg} / \mathrm{dl}$ were accepted as hypocalcemia. Acute hypocalcemia may lead to cardiovascular effects such as syncope, congestive heart failure, neuromuscular such as drowsiness and tingling sensation in the perioral region or fingers, carpopedal spasm, tetani, wheezing, bronchospasm and laryngospasm, and neurologic symptoms such as irritability, personality changes may occur. Patients with hypocalcemia and clinical findings were treated with oral calcium \pm vitamin $\mathrm{D}$ preparations and intravenous calcium gluconates. Patients who need calcium replacement treatment more than 6 months were accepted as permanent hypocalcemia, others were accepted as transient hypocalcemia. All hypocalcemic patients were consulted with the Endocrinology Department and the permanent ones were taken under surveillance of the Endocrinology Department.

Surgical site controls were done at the end of first week. Patients having shortness of breath or dyspnea were examined immediately after surgery but asymptomatic patients were examined on postoperative $2 \mathrm{nd}$ day for laryngeal functions. Patients with vocal cord paralysis were followed periodically at $1,2,3,6,12$, and 24 months after the surgery. Permanent RLN paralysis was accepted if the vocal cord functions did not resolve by the end of the 1st year follow-up. Beginning of the mobility in flexible fiberoptic laryngoscopy and resolution of symptoms were recorded in transient vocal cord paralysis.

All patients were operated under general anesthesia with endotracheal intubation. Sevoflorane was used for maintenance treatment in both groups and additional muscle relaxants (rocuronium) were used only patients in group 2. 


\section{Operative technique}

Patients were operated under semi-fowler position, low collar skin incision was performed, and subcutaneous tissue and platysma muscle were dissected. Strep muscles were longitudinally dissected and laterally retracted. Middle thyroid vein was ligated using LigaSure Precise ${ }^{\circledast}$ (LigaSure Vessel Sealing System, Medtronic, USA) and started to dissection of upper pole. In all patients, upper thyroid poles were clamped adjacent to the thyroid gland and ligated using no: $2 / 0$ silk sutures .

In all patients, RLN was dissected and visualized in tracheoesophageal groove. Parathyroid glands were found and preserved using LigaSure. Finally, tracheal ligamentous connections were resected, and hemithyroidectomy procedure was completed. Same procedures were performed for the other side, and total thyroidectomy was completed. In near-total thyroidectomy procedure, total hemithyroidectomy performed in one side, and following this, less than one-gram thyroid tissue is left behind and RLN was partially visualized. In addition, negative pressure hemovac drains were used if necessary.

All thyroid surgeries in both groups were performed under general anesthesia. In Group 1, patients were operated using Nerve Integrity Monitor (Dr Langer Medical, Germany). An endotracheal tube with an integrated surface electrode was inserted between the vocal folds by an anesthesiologist under direct vision during intubation. Neuromonitorization started after resolution of the muscle relaxants' effect. Repetitive 1-mA-2-mA stimulation of the RLN was performed with a standard intermittent monopolar or bipolar probe. Proper stimulation was defined with both the audible alarm of the NIM system and observation of a recognizable EMG waveform ( $>100 \mu \mathrm{V}$ for RLN).

In Group 2, patients were operated and all RLNs were tried to be identified only with direct visualization.

\section{Statistical analysis}

SPSS 15.0 for Windows was used for statistical analysis. In descriptive statistics, the categorical variables were expressed as number and percentage and the numerical variables were expressed as mean, standard deviation, and median. If the numerical variables were normally distributed, Student's ttest was used to compare two independent groups. If the numerical variables were not normally distributed, Man$\mathrm{n}$-Whitney U-test was used to compare two independent groups. Chi-square test was used to compare ratios in groups. $\mathrm{P}<0.05$ was considered statistically significant.

\section{RESULTS}

Of 212 patients, complete data, which fit the inclusion criteria of this study, was found in 191 patients. Group 1 comprised 79 patients and Group 2 comprised 112 patients. M/F ratio was $72 / 7$ in Group 1 and 102/10 in Group 2. In the comparison of the groups with demographic parameters; mean age in Group 1 was $49.28 \pm 11.419$ and in Group was 47.11 \pm 11.994 . Mean BMI of the patients in Group 1 was 26.8965 and in Group 2 was 26.6905. According to these two parameters, there is no significant difference between the groups (age, p:0.210; BMI, p:0.684).

Surgical procedures and number of nerves under the risk (NUR) are presented in Table 1.

Operation time was defined as the duration starting from skin incision and lasting to the end of the skin closure. Operation time was found to be significantly shorter in Group 1 (Mean time, $93.08 \mathrm{~min}$ ) than in Group 2 (Mean time, $116.54 \mathrm{~min})(\mathrm{p}=0.03)$.

Pathologic results included benign nodule, cyst, adenoma, Hashimato's thyroiditis, lymphocytic thyroiditis, and papillary carcinoma.

Impairment in vocal cord mobility unilaterally was observed in 7 patients in Group 1 (8.9\%) and 15 patients in Group 2 (13.4\%) on the postoperative 1st day. According to the statistical tests, we did not find significant difference between the groups $(p=0.368)$. In Group 1, vocal cord paralysis and clinical symptoms recovered in 3 patients at 1 st month control, in 1 patient at $3 \mathrm{rd}$ month control, and in 1 patient at 6th month control; these 5 patients were accepted as transient RLN paralysis. Only

TABLE 1. Surgical procedures and the number of nerves under the risk (NUR)

\begin{tabular}{ccc} 
Group 1 & Patients $(\mathrm{n})$ & NUR \\
\hline Total Thyroidectomy & 42 & 84 \\
Near-Total Thyroidectomy & 32 & 64 \\
Hemithyroidectomy & 5 & 5 \\
Total & 79 & 153
\end{tabular}

Group 2
Total Thyroidectomy

Near-Total Thyroidectomy

Hemithyroidectomy

Total

Total

46

59

7

112

191
92

118

7

217

370 
TABLE2. Distribution of complications in the groups

\begin{tabular}{lccc} 
& $\begin{array}{c}\text { Group 1 } \\
\mathrm{n}(\%)\end{array}$ & $\begin{array}{c}\text { Group 2 } \\
\mathrm{n}(\%)\end{array}$ & $\mathrm{p}$ \\
\hline Transient VC paralysis & $5(3.26)$ & $11(5.06)$ & $\mathrm{NS}$ \\
Permanent VC paralysis & $2(1.30)$ & $4(1.84)$ & $\mathrm{NS}$ \\
Transient hypocalcemia & $4(5.06)$ & $14(12.5)$ & NS \\
Permanent hypocalcemia & $1(1.26)$ & $4(3.57)$ & NS \\
Hematoma & $1(1.26)$ & $3(2.67)$ & NS \\
Suture reaction & $2(2.53)$ & $3(2.67)$ & NS
\end{tabular}

two patients did not show any improvement or recovery in the first year control so they were accepted as permanent RLN paralysis. In Group 2, vocal cord paralysis and clinical symptoms recovered in 7 patients at 1 st month control, in 2 patients at 3rd month control, and in 2 patients at 6th month; these 11 patients were accepted as transient RLN paralysis. Four patients did not show any improvement or recovery in 1st year control, so they were accepted as permanent RLN paralysis (Table 2). We did not perform any vocal cord surgery for permanent paralytic patients.

Both permanent and transient hypocalcemia was encountered in 5 patients (6.3\%) in Group 1, 18 patients $(16.1 \%)$ in Group 2; this difference was statistically significant $(p=0.045)$. Four of the 5 patients recovered during follow-up period, only 1 of them was accepted as permanent in Group 1. Of 18 patients, 14 recovered during follow-up period and were accepted as transient hypocalcemia and 4 were accepted as permanent hypocalcemia.

Hematoma was encountered in one patient $(1.3 \%)$ in Group 1 and in three patients (2.7\%) in Group 2. Two patients in Group 2 and one patient in Group 1 were reoperated on postoperative 1st day, and bleeding control was achieved under the general anesthesia. Other patient in Group 2 had relatively minor bleeding and was followed closely without reoperation. Suture reaction was encountered in two patients $(2.5 \%)$ in Group 1 and in three patients $(2.7 \%)$ in Group 2 . There is no significant difference between the groups according to these complication rates (hematoma $\mathrm{p}=0.796$, suture reaction $\mathrm{p}=0.796$ ).

In our case series, we did not encounter bilateral vocal cord paralysis, necessity of intensive care, and mortality. According to hospitalization of patients there is no significant difference between two groups (in Group 1, $1.07 \pm 0.27$ days; in Group 2, $1.06 \pm 0.28$ days). Mean recovery time for transient paralysis in Group 1 was 8 weeks (3-18 weeks) and in Group 2 was 9 (3-19) weeks.Cessa- tion of calcium replacement therapy in transient hypocalcemic patients in Group 1 was 5 weeks (3-9) and in Group 2 was 5 weeks (3-9).

\section{DISCUSSION}

RLN injury is the most scary and serious complication because of the decline in quality of life and labor loss [6, $15]$, but hypocalcemia is the most frequent complication in thyroid surgery $[16,17]$. In this study we aimed to investigate the effect of IONM usage on thyroidectomy complications. In literature, the incidence of RLN paralysis varies with a wide range of $0-26 \%[1-3,5,18,19]$. Fortunately, most of these RLN paralyses are transient, and the incidence of permanent paralysis varies with a range of $0-5.8 \%$ in literature $[5,6,20,21]$. Permanent paralysis is accepted when the nonfunctioning or dysfunctioning of the vocal cord lasts for more than 1 year [3]. This study presented the median recovery time for transient RLN paralysis as 8 (4-12) weeks, and in some instance it may last up to 12 months [3]. Joliat et al. [3] reported the incidence of RLN paralysis in their case series including 456 thyroidectomy as $14 \%$; in addition, they encountered 8 cases of paralyses even with IONM usage. In this study, the incidence of permanent paralysis is $1.1 \%$. In another more recent study with 5252 cases series, permanent paralysis incidence was reported to be 1.2\% [4].

Dissection and identification of RLN may be problematic in reoperation, malignancy, anatomic distortion due to huge sized tumors, inflammation, and radiation history [1]. In these situations, IONM usage is strictly advised by many authors $[7,15]$. Wojtczak et al. [10] reported the incidence of transient and permanent paralysis in recurrent thyroid surgery as $12.5 \%$ and 3.8\%, respectively. Hermann et al. [2] reported the incidence of permanent RLN paralysis if the nerve just localized as $0.9 \%$, if partially dissected as $0.3 \%$, if totally dissected as $0.1 \%$. Therefore, they advocate that the detailed and careful dissection of RLN significantly decrease the risk of RLN injury.

After the introduction of IONM in 1990, improvement in RLN injury incidence is expected; however, there exists is a discrepancy about the results in literature $[4,10,19,22]$. Shindo $M$ et al. [23] reported in their study with 684 cases that IONM does not change the incidence of transient or permanent vocal cord paralysis. Calo et al. [24] did not show any significant difference between the direct visualization and IONM usage for incidence of either transient or permanent vocal cord 
paralysis. Pisanu et al. [25] compared direct visual technique with IONM usage in their metaanalysis and analyzed 20 retrospective study with total 35513 nerves (24038 IONM and 11475 direct visualization) and did not find any significant difference in the incidence of RLN paralysis (IONM group, $3.47 \%$ and direct visualization, 3.67\%). Malik et al. [22] also found similar results in their systematic review. Yang et al. [9] found statistically significant decrease in transient and overall RLN paralysis incidence but did not find significant difference in permanent paralysis incidence. Rulli et al. [26] also reported similar results with Yang and associate this finding with higher frequency of transient paralysis and complete resolution in longer periods. Brajcich et al. [5], an experienced endocrine surgeon "in a high-volume thyroidectomy practice," stated that IONM usage is not a proven tool to decrease the RLN injury risk; however, in the case of lost signal of RLN in one side may be helpful to give up the otherside operation. In their retrospective study with 243.527 thyroidectomy cases, Chung et al. reported higher incidence of RLN paralysis in IONM patients. However, they attributed this finding with higher incidence of complications in the first year of IONM usage, and they also stated that this complication risk decreases by the higher experience of this device. In the subgroup analysis of this study, IONM usage significantly decreased the risk of RLN injury in total thyroidectomy patients with neck dissection [19].

To identify and predict postoperative vocal cord functions, intermittent-type IONM is frequently used; however, in recent years, continuous-type IONM usage is getting popular $[4,5,8]$. In their study with 195 caes with vagal nerve stimulation, Farizon et al. reported the incidence of transient RLN paralysis as $3.1 \%$ and incidence of permanent RLN paralysis as $0.51 \%$. In this study, they prefer to use the bipolar trans-cricothyroid ligament electrode and indirect stimulation of RLN with vagal nerve stimulation [27]. However, unfortunately, necessity of carotid sheat dissection, difficulty of electrode implantation in complex cases, potential side effects of vagal nerve stimulation, and cost effectiveness are the limitation factors of continuous-type IONM usage [20]. Liu et al. in their study used standard intermittent IONM in thyroid surgeries with 208 nerves under risk. Of 19 cases with 50\% loss of EMG amplitude, they stopped surgical procedure, prevented the total signal loss, and finally experienced only two transient paralyses with 2 months of recovery time [20]. In our series, according to NUR, the incidence of transient paralysis was $4.32 \%$ and that of permanent paralysis was $1.62 \%$ (Table 2), which is consistent with literature. In our study, we did not find significant advantages of intermittent-type IONM usage in the prevention of RLN injury.

In our study, we found significantly shorter operation time in Group 1. Similarly, Sar1 et al. found significantly shorter duration of operation in IONM group in their study. They did not find significant difference according to incidence of RLN paralysis but they advocated that IONM usage significantly decreases the identification time of RLN [1]. However, using neuromonitoring in thyroid surgery is related with increased preoperative setup time [23]. However, total operative time was not significantly different between the groups in metaanalysis [25]. According to these results, the shortness of the operation in Group 1 seems as a secondary advantage of IONM usage.

Hypocalcemia is the most frequent complication of thyroidectomy. The most important factors influencing postoperative development of hypocalcemia include intraoperative trauma to parathyroid gland or its vasculature, inability to identify parathyroid gland during operation, incidental parathyroidectomy, central dissection, bilateral thyroidectomy, and inadequate experience of the surgeon $[6,16,28]$. Luo et al. [30] described symptomatic hypocalcemia as specific numbness, spasm, muscular cramp, and Chevok's syndrome. Again, in this study, the incidence of biochemical hypocalcemia was $26.97 \%$ and symptomatic hypocalcemia was $12.5 \%$. The rates of temporary and permanent hypocalcemia in the literature are widely distributed with 1.6\%-50\% and $0.7 \%-4.8 \%$, respectively $[6,16,28,29]$. These widely-distributed prevalence of hypocalcemia is mostly related with uncertainty on the definition of hypocalcemia, different dissection technique, and variations in calcium and vitamin D replacement treatment plan [30]. In that study, they found female gender, hypomagnesia, and lower level of intact PTH as the risk factors for hypocalcemia. In addition to female gender and lower levels of intact PTH levels, Noureldine et al., [16] in their study with 304 total thyroidectomy patients, found malignancy as an independent predictive factor for hypocalcemia. In our study, according to the hypocalcemia complication rates, we found significantly lower hypocalcemia in Group 1. We did not perform any different techniques to the groups for parathyroid preservation; however, it was significantly lower in Group 1. This may be incidental or may be related with giving relatively more attention to the adjacent tissues in Group 1. Again, early and easy identification of RLN gives more confidence to surgeon and decreases the stress level $[1,24]$. We suggest that this easy identification of RLN is a major factor for significant hypocalcemia in Group 1.

In our study, we did not find any significant difference between the groups for hematoma incidence (Group1, 1.26\%; Group 2, 2.67\%). In literature, we found hematoma incidence to be $1.2 \%-1.3 \%$ in thyroidectomies $[6,16,29]$. 


\section{CONCLUSION}

Our series and similar studies in literature did not reveal the benefits of intermittent IONM usage for decreasing the rates of RLN injury. However, we found significant decrease in operation time and hypocalcemia rates. Increasing the confidence of surgeon by easy identification of RLN may be a factor for lower hypocalcemia rates. Wider prospective studies and series may be helpful for understanding the effect of IONM on RLN injury and other complications.

Ethics Committee Approval: This retrospective study was approved with local Ethic Committee of Haseki Training and Research Hospital, Istanbul-Turkey (Date/No:10.08.2016/366).

Conflict of Interest: No conflict of interest was declared by the authors.

Financial Disclosure: The authors declared that this study has received no financial support.

Authorship contributions: Concept - S.D., T.D., E.C.; Design S.D., T.D., E.C.; Supervision - S.D., T.D., E.C.; Materials - S.D., T.D., E.C.; Data collection \&/or processing - S.D., T.D., E.C.; Analysis and/ or interpretation - S.D., T.D., E.C.; Writing - S.D., T.D., E.C.; Critical review - S.D., T.D., E.C.

\section{REFERENCES}

1. Sar1 S, Erbil Y, Sümer A, Agcaoglu O, Bayraktar A, Issever H, et al. Evaluation of recurrent laryngeal nerve monitoring in thyroid surgery. Int J Surg 2010;8:474-8. [CrossRef]

2. Hermann M, Alk G, Roka R, Glaser K, Freissmuth M. Laryngeal recurrent nerve injury in surgery for benign thyroid diseases: effect of nerve dissection and impact of individual surgeon in more than 27,000 nerves at risk. Ann Surg 2002;235:261-8. [CrossRef]

3. Joliat GR, Guarnero V, Demartines N, Schweizer V, Matter M. Recurrent laryngeal nerve injury after thyroid and parathyroid surgery: Incidence and postoperative evolution assessment. Medicine (Baltimore) 2017;96:e6674.

4. Bergenfelz A, Salem AF, Jacobsson H, Nordenström E, Almquist M; Steering Committee for the Scandinavian Quality Register for Thyroid, Parathyroid and Adrenal Surgery (SQRTPA). Risk of recurrent laryngeal nerve palsy in patients undergoing thyroidectomy with and without intraoperative nerve monitoring. Br J Surg 2016;103:1828-38. [CrossRef]

5. Brajcich BC, McHenry CR. The utility of intraoperative nerve monitoring during thyroid surgery. J Surg Res 2016;204:29-33. [CrossRef]

6. Karamanakos SN, Markou KB, Panagopoulos K, Karavias D, Vagianos $\mathrm{CE}, \mathrm{Scopa} \mathrm{CD}$, et al. Complications and risk factors related to the extent of surgery in thyroidectomy. Results from 2,043 procedures. Hormones (Athens) 2010;9:318-25. [CrossRef]

7. Wong KP, Mak KL, Wong CK, Lang BH. Systematic review and metaanalysis on intra-operative neuro-monitoring in high-risk thyroidectomy. Int J Surg 2017;38:21-30. [CrossRef]

8. Anuwong A, Lavazza M, Kim HY, Wu CW, Rausei S, Pappalardo V, et al. Recurrent laryngeal nerve management in thyroid surgery: consequences of routine visualization, application of intermittent, standardized and continuous nerve monitoring. Updates Surg 2016;68:331-41. [CrossRef]

9. Yang S, Zhou L, Lu Z, Ma B, Ji Q, Wang Y. Systematic review with metaanalysis of intraoperative neuromonitoring during thyroidectomy. Int $\mathrm{J}$ Surg 2017;39:104-13. [CrossRef]

10. Wojtczak B, Barczyński M. Intermittent neural monitoring of the recurrent laryngeal nerve in surgery for recurrent goiter. Gland Surg 2016;5:481-9.

11. Lin HS, Terris DJ. An update on the status of nerve monitoring for thy$\mathrm{roid} /$ parathyroid surgery. Curr Opin Oncol 2017;29:14-9. [CrossRef]

12. Chandrasekhar SS, Randolph GW, Seidman MD, Rosenfeld RM, Angelos P, Barkmeier-Kraemer J, et al; American Academy of Otolaryngology-Head and Neck Surgery. Clinical practice guideline: improving voice outcomes af- ter thyroid surgery. Otolaryngol Head Neck Surg 2013;148:S1-37.

13. Randolph GW, Dralle H; International Intraoperative Monitoring Study Group, Abdullah H, Barczynski M, Bellantone R, et al. Electrophysiologic recurrent laryngeal nerve monitoring during thyroid and parathyroid surgery: international standards guideline statement. Laryngoscope 2011;121 Suppl 1:S1-16. [CrossRef]

14. Musholt TJ, Clerici T, Dralle H, Frilling A, Goretzki PE, Hermann MM, et al; Interdisciplinary Task Force Guidelines of the German Association of Endocrine Surgeons. German Association of Endocrine Surgeons practice guidelines for the surgical treatment of benign thyroid disease. Langenbecks Arch Surg 2011;396:639-49. [CrossRef]

15. Gardner IH, Doherty GM, McAneny D. Intraoperative nerve monitoring during thyroid surgery. Curr Opin Endocrinol Diabetes Obes 2016;23:394-9. [CrossRef]

16. Noureldine SI, Genther DJ, Lopez M, Agrawal N, Tufano RP. Early predictors of hypocalcemia after total thyroidectomy: an analysis of 304 patients using a short-stay monitoring protocol. JAMA Otolaryngol Head Neck Surg 2014;140:1006-13. [CrossRef]

17. Inversini D, Rausei S, Ferrari CC, Frattini F, Anuwong A, Kim HY, et al. Early intact PTH (iPTH) is an early predictor of postoperative hypocalcemia for a safer and earlier hospital discharge: an analysis on 260 total thyroidectomies. Gland Surg 2016;5:522-8. [CrossRef]

18. Jeannon JP, Orabi AA, Bruch GA, Abdalsalam HA, Simo R. Diagnosis of recurrent laryngeal nerve palsy after thyroidectomy: a systematic review. Int J Clin Pract 2009;63:624-9. [CrossRef]

19. Chung TK, Rosenthal EL, Porterfield JR, Carroll WR, Richman J, Hawn MT. Examining national outcomes after thyroidectomy with nerve monitoring. J Am Coll Surg 2014;219:765-70. [CrossRef]

20. Liu XL, Wu CW, Zhao YS, Wang T, Chen P, Xin JW, et al. Exclusive real-time monitoring during recurrent laryngeal nerve dissection in conventional monitored thyroidectomy. Kaohsiung J Med Sci 2016;32:135-41.

21. Randolph GW, Kamani D. Intraoperative electrophysiologic monitoring of the recurrent laryngeal nerve during thyroid and parathyroid surgery: Experience with 1,381 nerves at risk. Laryngoscope 2017;127:280-6.

22. Malik R, Linos D. Intraoperative Neuromonitoring in Thyroid Surgery: A Systematic Review. World J Surg 2016;40:2051-8. [CrossRef]

23. Shindo M, Chheda NN. Incidence of vocal cord paralysis with and without recurrent laryngeal nerve monitoring during thyroidectomy. Arch Otolaryngol Head Neck Surg 2007;133:481-5. [CrossRef]

24. Calò PG, Pisano G, Medas F, Tatti A, Pittau MR, Demontis R, et al. Intraoperative recurrent laryngeal nerve monitoring in thyroid surgery: is it really useful? Clin Ter 2013;164:e193-8.

25. Pisanu A, Porceddu G, Podda M, Cois A, Uccheddu A. Systematic review with meta-analysis of studies comparing intraoperative neuromonitoring of recurrent laryngeal nerves versus visualization alone during thyroidectomy. J Surg Res 2014;188:152-61. [CrossRef]

26. Rulli F, Ambrogi V,Dionigi G, Amirhassankhani S, Mineo TC, Ottaviani F, et al. Meta-analysis of recurrent laryngeal nerve injury in thyroid surgery with or without intraoperative nerve monitoring. Acta Otorhinolaryngol Ital 2014;34:223-9.

27. Farizon B, Gavid M, Karkas A, Dumollard JM, Peoc'h M, Prades JM. Intraoperative monitoring of the recurrent laryngeal nerve by vagal nerve stimulation in thyroid surgery. Eur Arch Otorhinolaryngol 2017;274:421-6.

28. Ozemir IA, Buldanlı MZ, Yener O, Leblebici M, Eren T, Baysal H, et al. Factors affecting postoperative hypocalcemia after thyroid surgery: Importance of incidental parathyroidectomy. North Clin Istanb 2016;3:9-14.

29. Rosato L, Avenia N, Bernante P, De Palma M, Gulino G, Nasi PG, et al. Complications of thyroid surgery: analysis of a multicentric study on 14,934 patients operated on in Italy over 5 years. World J Surg 2004;28:271-6.

30. Luo H, Yang H, Zhao W, Wei T, Su A, Wang B, et al. Hypomagnesemia predicts postoperative biochemical hypocalcemia after thyroidectomy. BMC Surg 2017;17:62. [CrossRef] 